\title{
FRANÇOIS LECERCLE, L'œil oblique. Essais sur l'image, la peinture et le théâtre
}

\section{Luana Doni}

\section{(2) OpenEdition \\ Journals}

\section{Edizione digitale}

URL: https://journals.openedition.org/studifrancesi/45665

DOI: $10.4000 /$ studifrancesi.45665

ISSN: 2421-5856

\section{Editore}

Rosenberg \& Sellier

\section{Edizione cartacea}

Data di pubblicazione: 1 août 2021

Paginazione: 424-425

ISSN: 0039-2944

\section{Notizia bibliografica digitale}

Luana Doni, «FRAnçoIs LECERCLE, L'œil oblique. Essais sur l'image, la peinture et le théâtre», Studi Francesi [Online], 194 (LXV | II) | 2021, online dal 01 septembre 2021, consultato il 15 octobre 2022. URL: http:// journals.openedition.org/studifrancesi/45665 ; DOI: https://doi.org/10.4000/studifrancesi.45665

Questo documento è stato generato automaticamente il 15 octobre 2022.

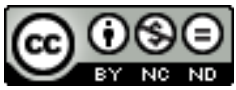

Creative Commons - Attribuzione - Non commerciale - Non opere derivate 4.0 Internazionale - CC BYNC-ND 4.0

https://creativecommons.org/licenses/by-nc-nd/4.0/ 


\title{
FRANÇOIS LECERCLE, L'œil oblique. Essais sur l'image, la peinture et le théâtre
}

\author{
Luana Doni
}

\section{NOTIZIA}

FRANÇOIS LECERCLE, L'œil oblique. Essais sur l'image, la peinture et le théâtre, Genève, Droz, 2020, 564 pp.

1 Il volume si concentra sugli scritti di FRANçOIS LECERCLE in merito al fenomeno interpretativo legato alla ricezione dell'opera d'arte. In particolare, il lavoro sembra essere volto allo studio di ciò che è, come egli stesso lo definisce, surrettizio all'interno del testo o dell'immagine. Ecco che la definizione di ceil oblique sta ad indicare la metodologia con la quale l'autore tenta di restituire il senso dell'opera d'arte attraverso uno sguardo svincolato da qualsivoglia teoria preconcetta; lo scopo di Lecercle non è quello di servirsi di un testo al fine di illustrare un'idea concepita aprioristicamente, ma di esaminare microscopicamente l'opera e prepararsi agli scenari inediti offerti dall'interpretazione. Questo tipo di pensiero, spiega Lecercle, non è mai astratto, o meglio, non lo è alla maniera del filosofo; il ricercatore, infatti, fonde la sua elaborazione teorica al concreto: un'immagine, un aneddoto, forniscono materiale utile alla formulazione del pensiero.

2 Gli articoli presenti nella raccolta cercano di dare risposta a tutte quelle domande che, negli anni, sono risultate essere alla base del lavoro di Lecercle: la pittura, il teatro, l'esegesi, quelle discipline che hanno in comune il fatto di non servirsi di uno sguardo diretto, ma di necessitarne uno furtivo, dietro le quinte.

3 Il primo articolo della raccolta (Donner à ne pas voir) si concentra sulla convinzione che, nonostante sembri paradossale data la sua stessa natura, la pittura non faccia che mostrare la stessa quantità di cose che, al contempo, nasconde. Il grande artista diventa, così, colui che mostra l'osceno: il sangue sotto la pelle, il corpo sotto i vestiti, ma che procede, contemporaneamente, a far arretrare la frontiera del visibile, a 
illudere l'occhio, a ingannarlo. Il paradosso è spiegato da Lecercle attraverso due aneddoti celebri, due storie di rivalità, quella tra Zeusi e Parrasio e quella tra Apelle e Protogene, narrate da Plinio il Vecchio.

4 Il secondo articolo (La laideur de Giotto), invece, prende in esame una breve novella del Decamerone, quella che vede protagonista il pittore Giotto, al fine di dimostrare come la letteratura sia in grado di dimostrare il surrettizio della pittura. La novella dichiara la superiorità dell'artigiano/artista sull'intellettuale, nobilitando la figura del pittore e, implicitamente, tentando di riconoscere le nuove ambizioni delle arti figurative.

Il terzo articolo, in chiusura della prima sezione del volume, indaga sulla questione dell'uso dell'oro nella pittura. In ambito artistico, l'oro dà origine a un doppio discorso: religioso ed economico. Quest'ultimo mina la sacralità dell'arte, facendo dell'uso dell'oro caratteristica fondamentale nella stima del valore dell'opera.

6 La seconda sezione di questa prima parte della raccolta («Nature mortes et vanités: Chardin, Damien Hirst») si focalizza sul lavoro dei due artisti, Chardin e Damien Hirst, tanto lontani nel tempo eppure avvicinabili per certi aspetti del loro sguardo. In merito a Chardin, pittore celebre per le sue nature morte, l'autore si interroga sulla possibilità di riflessione generata dalla particolare scelta dei soggetti. L'opera di Chardin ha il merito di incarnare tutte le contraddizioni della sua epoca; La Raie simboleggia perfettamente l'ultimo grado dell'abiezione. La spiegazione data al successo di Chardin in ambito aristocratico è quella di aver epurato la bassezza quotidiana e, come sostiene Diderot, di aver realizzato un doppio scopo, quello di aver invertito la repulsione in fascinazione e l'handicap in trionfo dell'arte.

7 Per tutto il corso della storia, la vanitas, in pittura, si è sempre interfacciata con un duplice paradosso: il fatto di riuscire a far provare piacere per quelle immagini che, abitualmente, nella realtà, suscitano disgusto, e il fatto di imbellire l'orrido per mettere in evidenza la convenzionale bellezza e condannarla. Emblema di questa particolare arte pittorica è Damien Hirst, l'enfant terrible dell'arte britannica. La sua opera si presenta in realtà come vanitas moderna; ne è un esempio la scultura For the Love of God, un cranio umano cosparso di diamanti in rappresentanza sì di un significato spirituale simboleggiato dalla mortalità esibita dal cranio, ma anche della mondanità, la presenza della materialità ostentata dai diamanti. L'autore non manca di fare della sua arte una provocazione religiosa; Jesus and the Disciples esibisce in un'unica installazione dodici bacheche contenenti ciascuna una testa di mucca, una sola bendata in rappresentanza di Giuda, e una tredicesima bacheca contenente del liquido. L'opera di Hirst si differenzia dalla vanitas tradizionale proprio a causa di quel gusto per la provocazione e di quella componente ludica che contraddistingue opere come For the Love of God, tra le più costose nel mercato dell'arte a causa del faraonico valore delle sue componenti. La questione finanziaria intorno al cranio di Hirst è responsabile di una conversione dell'arte in marketing e dell'opera in oggetto pubblicitario, una bolla speculativa.

8 La seconda parte del volume («Théologie des images et exégèse») è concentrata sugli articoli pubblicati da Lecercle intorno agli anni Ottanta e che indagano i rapporti tra pittura e letteratura.

9 La questione del piacere dell'immagine è il primo tema ad essere affrontato dallo studioso, che prende in esame l'arco temporale 1550-1650, quando il piacere era identificato come scopo dell'arte. È proprio intorno alla questione del piacere che teoria sacra e profana si scontrano; per quanto riguarda la teoria profana, l'idea che l'arte debba abbandonare la voluttà è messa in funzione della tradizione di stampo 
retorico, che vede nel piacere sensuale una percezione ad appannaggio esclusivo degli ignoranti. I teorici sacri, benché in sintonia con il pensiero profano per quanto riguarda l'abbandono del piacere sensuale nella percezione artistica, rafforzano alcune posizioni di diffidenza nei confronti della questione. In ambito sacro, la prevalenza dell'aspetto sensoriale nell'arte rappresenta un doppio rischio: quello della perdita del pubblico religioso e il rischio di trattare alcuni soggetti biblici in maniera eccessivamente sensuale.

10 Successivamente, Lecercle passa ad elencare le strategie di riabilitazione dell'arte, passando da quella sociale alla concezione di piacere spirituale attraverso un processo traslativo, per poi approfondire la controversa questione dell'idolatria, del passaggio dalla reliquia all'immagine e all'enigma verbale nella resa figurativa.

11 La terza e ultima parte del volume («Art de la scène») prende come oggetto di studio le arti sceniche. Gli articoli di Lecercle rivolti al teatro vengono divisi in due blocchi: Théâtre et vision e La scène et l'obscène.

12 Lecercle traccia i punti di convergenza tra la scena pittorica e quella teatrale. In particolare, riportando l'esempio di quello che accadde per il Véritable Saint Genest in cui la componente scenografica dello spettacolo, il décor, è parte attiva della rappresentazione, contrariamente a quanto accadeva nel teatro elisabettiano, dove l'assenza di scenografia costringeva gli interpreti ad usare la parola in funzione descrittiva dello spazio.

Nel teatro francese, dunque, l'attenzione dedicata al décor è indiscutibilmente importante, anche e soprattutto per quanto riguarda lo spettatore. L'avvicinamento di Lecercle al teatro è volto in particolare a ciò che lo spettacolo non mostra; Lecercle spiega che il teatro non si limita a rappresentare le immagini, bensì a produrle in maniera inedita e proibita. Una drammaturgia invisibile, come la definisce lui, che lascia al lettore/spettatore la capacità di immaginare la violenza, l'amore, la gioia o il tacito e perverso piacere provato da Medea nella lentezza del suo gesto, che va oltre l'infanticidio. 